\title{
Réparation des dommages pour violation de confiance et attentes légitimes : une analyse des systèmes juridiques Français et Colombien*
}

\author{
[Artículos]
}

\author{
Ciro Nolberto Güechá Medina ** \\ Jessica Tatiana Güechá Torres****
}

Fecha de Recepción: 4 de marzo de 2021

Fecha de Aprobación: 18 de mayo de 2021

Citar como:

Güechá Medina, C. N. y Güechá Torres, J. T. (2021). Réparation des Dommages pour Violation de Confiance et Attentes Légitimes : Une Analyse des Systèmes Juridiques Français et Colombien. Via Inveniendi Et Iudicandi, 16(2). https://doi.org/10.15332/19090528.6782

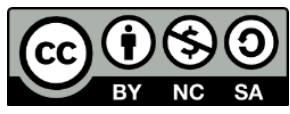

\section{Résumé}

La confiance légitime implique la croyance ou la conviction intime qu'une procédure administrative sera maintenue sans changement ni aucune surprise, pour atteindre les objectifs visés ; cette circonstance génère des

\footnotetext{
* El artículo es producto del proyecto de investigación "La incidencia de la potestad discrecional administrativa en la gestión judicial", gestionado en la Universidad Santo Tomás, sede Bogotá, Colombia.

** Avocat, Doyen émérite de la Faculté de Droit I'Université Santo Tomás de Colombie, Docteur en droit, Docteur en Droit Public et Docteur en Philosophie. Correo electrónico: ciroguecha@hotmail.com; ORCID: https://orcid.org/0000-0003-1462-4853

*** Avocat de I'Université du Rosario, Master en Droit Public, Diplôme d'Études Supérieures de I'Université de Paris 2 Panthéon Assas, Master II de Droit Public Approfondi de I'Université de Paris 2 Panthéon Assas. Correo electrónico: tatiana-guecha@hotmail.com; ORCID:

https://orcid.org/0000-0002-4298-9182
}

Via Inveniendi Et Iudicandi

e-ISSN: 1909-0528 | DOI: https://doi.org/10.15332/19090528

Vol. 16 N.o 2 | julio-diciembre del 2021 
attentes légitimes en ce qui concerne la probabilité d'atteindre un droit ; c'est-à-dire pour obtenir un droit futur ou en cours de consolidation.

Dans cette mesure, la confiance et les attentes légitimes sont parfois déçues, produisant des préjudices patrimoniaux aux personnes qui interviennent dans la procédure administrative et générant ce que le système juridique colombien a appelé un dommage illicite, c'est-à-dire indemnisable.

Cette circonstance permet d'analyser le mode de fonctionnement de la responsabilité de l'État en France et en Colombie, lorsqu'interviennent des dommages ou des pertes indemnisables. Cela se concrétise dans la reconnaissance des droits futurs ou en cours de consolidation, ou dans les compensations économiques correspondantes.

Mots clés : Confiance, attente légitime, responsabilité, dommage de l’État, dommage, réparation.

\section{Reparación de daños por violación de la confianza y esperanzas legítimas: un análisis de los sistemas jurídicos Francés y Colombiano}

\section{Resumen}

La confianza legítima implica la creencia o la convicción íntima de que un procedimiento administrativo puede mantenerse sin cambios ni sorpresas para lograr sus objetivos. Esta circunstancia genera esperanzas legítimas con respecto a la posibilidad de obtener un derecho, es decir, un derecho futuro o en curso de consolidación.

En esta medida, la confianza y las esperanzas legítimas se ven a veces defraudadas, provocan perjuicios patrimoniales a las personas que intervienen en el procedimiento administrativo y generan lo que el sistema jurídico colombiano llama un daño antijurídico, es decir, indemnizable.

Esta circunstancia permite analizar el funcionamiento de la responsabilidad del Estado en Francia y en Colombia cuando se

Via Inveniendi Et Iudicandi

e-ISSN: 1909-0528 | DOI: https://doi.org/10.15332/19090528

Vol. 16 N.० 2 | julio-diciembre del 2021 
producen daños o perjuicios indemnizables. Esto se materializa en el reconocimiento de los derechos futuros o en curso de consolidación o en las compensaciones económicas correspondientes.

Palabras claves: confianza, esperanza legítima, responsabilidad, daño estatal, daño, reparación.

\section{Repair of damages for breach of trust and legitimate expectations: an analysis of the French and Colombian legal systems}

\section{Abstract}

Legitimate expectation implies the belief or intimate conviction that an administrative procedure can be maintained without any changes or surprises in order to achieve its objectives. This circumstance generates legitimate expectations regarding the likelihood of obtaining a right, that is, a future right or a right in the process of consolidation.

To this extent, trust and legitimate expectations are sometimes frustrated, causing pecuniary damage to those involved in the administrative procedure and generating what the Colombian legal system has called unlawful damage, that is, that can be compensated. This circumstance makes it possible to analyze the functioning of the State's liability in France and Colombia, when damages that can be compensated occur. This is reflected in the recognition of future rights or in the process of consolidation or in the corresponding economic compensations.

Keywords: trust, legitimate expectation, liability, responsibility, state damage, damage, reparation.

\section{Introduction}

Les notions de confiance et d'attentes légitimes sont particulièrement liées, au point que, dans certains cas, elles sont traitées de la même manière. 
Toutefois, quoi qu'il arrive, la violation de cette confiance ou de ces attentes peut générer des dommages aux individus, qui doivent être indemnisés par l'administration, dans la mesure où la personne qui en souffre n'est pas obligée de résister générant ce que la loi colombienne appelle dommages illégaux.

Les critères de confiance et de confiance légitime ont connu une évolution particulière dans les systèmes juridiques français et colombien ; une circonstance qui se reproduit pour ce qui est de la réparation de dommages illicites causés par la violation du système.

Il convient de poser question suivante : Comment la réparation de dommages causés par la violation de la confiance et des attentes légitimes fonctionne-t-elle en France et en Colombie?

Le but à atteindre concerne la détermination du traitement accordé à la réparation des dommages causés par la violation de la confiance et des attentes légitimes en France et en Colombie. Pour cette raison compte tenu du fait qu'ils ont été considérés comme faisant partie de la reconnaissance des droits futurs ou en cours de consolidation et de la compensation financière suite aux pertes sur les capitaux propres.

Pour développer cette recherche, quelques hypothèses ont été posées :

- Les dommages et intérêts résultants de la violation de la confiance et des attentes légitimes impliquent la reconnaissance des droits futurs.

- La compensation financière constitue un mécanisme de réparation des dommages en cas de violation de la confiance et des attentes légitimes.

La méthodologie utilisée est de nature analytique propositionnelle, dans la mesure où les institutions de confiance et de confiance légitime sont analysées, ainsi que les formes de réparation des dommages, afin de déterminer la manière la plus appropriée pour que les droits des 
personnes puissent être protégés contre toute violation au cours de la procédure administrative.

\section{Développement}

\section{La Reconnaissance des droits futurs, une forme limitée face à la réparation des préjudices causés en France}

Il est évident qu'il y a des critères subjectifs qui doivent être pris en considération et que ce n'est pas seulement la loi au sens large qui régit l'activité étatique et surtout ses relations avec les particuliers dans le droit français.

Ainsi, à partir de principes tels que la sécurité juridique et de la reconnaissance expresse es attentes légitimes par le Conseil d'État et des attentes légitimes par le Conseil constitutionnel, l'importance acquise par l'individu dans le droit administratif et, en général, dans le droit public, est indéniable. En ce sens, et à partir des figures énoncées, il existe différentes formes de protection des intérêts particuliers.

En effet, à travers le principe de sécurité juridique, l'obligation d'adopter les mesures transitoires appropriées s'impose au pouvoir réglementaire et même au juge dans l'exercice de ses fonctions, lorsque leurs actes engendrent des affectations ou des charges excessives chez les destinataires, en vue de protéger les intérêts particuliers qui sont affectés par les mesures prises, comme c'est le cas des arrêts KPMG ou de la Société Techna (Calmes, 2015).

De même, lorsque le Conseil d’État reconnaît les espérances légitimes découlant des dispositions fiscales comme des biens, il s'agit d'une autre forme de garantie des intérêts particuliers dans la mesure où la haute juridiction concrétise les droits qui étaient en voie de consolidation, ce qui 
rentre dans le champ de protection de l'article 1 du protocole 1 de la Convention européenne des droits de l'homme.

Par exemple, dans l'arrêt Société EPI ou dans l'arrêt Société Vivendi, le Conseil d'État a annulé le refus de l'administration en ce qui concerne l'avantage fiscal que les Sociétés réclamaient sur la base du texte Initial. Cela a permis de reconnaitre le bénéfice demandé, se basant sur le fait que la nouvelle loi avait méconnue les espérances légitimes. De plus, l'intérêt général, pour lequel les lois avaient été adoptées, n'était pas suffisant pour justifier la limitation du droit de propriété, de sorte que la suppression des avantages fiscaux était disproportionnée. (Collet, 2017).

De plus, lorsqu'il adopte le concept des attentes légitimes du particulier comme étant une notion allant au-delà des droits acquis et des situations juridiques, le Conseil constitutionnel établit qu'un acte législatif ne peut méconnaître les effets légitimement fondés des situations légalement acquises et affirme la valeur de l'attente. Dans ce cas, si l'action du législateur n'est pas fondée sur un intérêt général suffisant, celle-ci peut être déclarée inconstitutionnelle ou le Conseil peut adopter des limites à l'application de la loi (Debré, 2014), c'est-à-dire que les dispositions favorables sont maintenues (Calmes, 2015).

Dans ces cas-là, on voit bien la façon dont les intérêts qui ne sont pas consolidés acquièrent une valeur juridique et bénéficient d'une protection dans le monde du droit. Néanmoins, il faut préciser que, si bien qu'il existe une action de l'État justifiée par des motifs d'intérêt général suffisamment pertinents pour limiter les droits tels qu’ils sont considérés par le Conseil d'État ou les attentes dans le cas du Conseil constitutionnel, normalement ceux-ci devraient être sacrifiés.

Toutefois, tel que l'annonce Camille Broyelle, il reste à savoir comment les hautes juridictions vont articuler les nouvelles règles de conduite face à 
l'activité du législateur, puisque celui-ci trouve de plus en plus de limites à l'exercice de ses fonctions, de sorte qu'il ne peut ignorer les espérances légitimes au regard des règles établies par la Convention européenne des droits de l'homme ou les situations légalement acquises et les effets que l'on peut attendre au regard des décisions du Conseil constitutionnel. Dès lors, si les actions du législateur sont contraires à ces postulats, il pourrait en résulter une responsabilité de l'État s'inscrivant dans le domaine de la faute (Broyelle, 2018).

Il s'agit là d'une position qui avait longtemps été écartée en raison du pouvoir souverain du législateur, ce qui impliquait que sa conduite était irréprochable (Truchet, 2019). Pourtant, le Conseil d'État a récemment prévu que la responsabilité de l'État pouvait être engagée en raison d'une loi contraire à la Constitution ou aux engagements internationaux (Conseil d'État, Requête no 425981 du 2019; Conseil d'État, Requête no 425983 du 2019 ; Conseil d'État, Requête $n^{\circ} 428162$ du 2019). C'était une décision attendue dans la mesure car, si bien des figures comme le contrôle de constitutionalité a posteriori des lois ont été introduites ; pour cette raison, il était inévitable que les dommages subis par une loi déclarée inconstitutionnelle ne soient pas reconnus.

Ainsi, le Conseil d'État distingue la réparation des préjudices découlant de l'adoption de la loi, événement dans lequel il est traité comme une responsabilité sans faute à cause de la rupture de l'égalité devant les charges publiques, de ceux dont les préjudices résultent de l'application de la loi, évènement dans lequel la responsabilité pourra être engagée en raison de l'omission des lois supérieures, c'est-à-dire une responsabilité pour faute.

En ce sens, la haute juridiction a indiqué que « la responsabilité de l'État peut être engagée en raison des exigences inhérentes à la hiérarchie des normes, pour réparer l'ensemble des préjudices qui résultent de

Via Inveniendi Et Iudicandi

e-ISSN: 1909-0528 | DOI: https://doi.org/10.15332/19090528

Vol. 16 N.० 2 | julio-diciembre del 2021 
l'application d'une loi méconnaissant la Constitution ou les engagements internationaux de la France ».

Il en est ainsi dans la mesure où celle-ci n'est pas susceptible d'être engagée :

[...] que si le Conseil constitutionnel a déclaré cette disposition inconstitutionnelle sur le fondement de l'article 61-1, lors de l'examen d'une question prioritaire de constitutionnalité, ou bien encore, sur le fondement de l'article 61, à l'occasion de l'examen de dispositions législatives qui la modifient, la complètent ou affectent son domaine. (Conseil d'État, Requête no ${ }^{4} 25981$ du 2019)

En outre, il faut :

[...] que la décision du Conseil Constitutionnel, qui détermine les conditions et les limites dans lesquelles les effets que la disposition a produits sont susceptibles d'être remis en cause, ne s'y oppose pas, soit qu'elle l'exclue expressément, soit qu'elle laisse subsister tout ou partie des effets pécuniaires produits par la loi qu'une action indemnitaire équivaudrait à remettre en cause. (Conseil d'État, Requête no 425981 du 2019)

Dans ces conditions, la victime affectée par les effets d'une loi contraire aux dispositions supérieures peut demander la réparation du préjudice, comme cela pourrait se produire dans les circonstances où une loi est déclarée inconstitutionnelle par la violation des situations légalement acquises et des effets légitimement attendus sans avoir une raison suffisante d'intérêt général.

Or, d'une manière générale, au-delà des notions qui ont été récemment développées par le Conseil d'État et le Conseil constitutionnel, il faut préciser que les espérances légitimes, en tant qu'intérêts allant au-delà des droits consolidés, si elles sont déçues, peuvent également trouver une 
protection à partir des formes classiques de responsabilité de l'État, de sorte qu'il est nécessaire d'établir la façon dont leur protection opère lorsque celles-ci se voient endommagées par l'action de l'administration ou même du législateur (Sänger, 2015)

De plus, la méconnaissance des espérances ou des attentes légitimes n'est pas non plus reconnue comme une forme indépendante génératrice de responsabilité, contrairement au système colombien où la méconnaissance des attentes légitimes sur la base du principe de confiance légitime engage la responsabilité de l'État (Conseil d'État Colombien, Arrêt no 22637 du 2015). La réparation des attentes ou des espérances qui ont été trompées est immergée dans la responsabilité pour faute et sans faute, au point que celles-ci peuvent être considérées comme étant un critère que le juge possède pour engager la responsabilité de l'État.

En effet, la responsabilité de l'État en droit français est considérée comme une grande conquête des citoyens (De Cupis, 1970, p. 81), car entre l'Ancien Régime et la première moitié du XIXe siècle, l'État était irresponsable des préjudices qu'il causait sur des fondements tels que la souveraineté, de sorte que « les préjudices éventuels étaient en quelque sorte des risques à courir par les citoyens » (Dupuis et Guédon, 2004, p. 555).

Toutefois, des exceptions, dans lesquelles l'État était tenu de réparer les dommages causés par son activité, sont progressivement prévues. Ce sont des exceptions qui sont devenues la règle à partir de principes tels que celui de légalité qui permettait de déduire si « une irrégularité entraînait une sanction, s'il y avait un dommage, donc une réparation » (Dupuis et Guédon, 2004, p. 555). Dans ce contexte, la protection des victimes contre les dommages subis par les actions de l'État était telle que la faute n'était plus nécessaire, de sorte que le dommage causé pouvait être réparé en raison du respect au principe d'égalité.

Via Inveniendi Et Iudicandi

e-ISSN: 1909-0528 | DOI: https://doi.org/10.15332/19090528

Vol. 16 N.० 2 | julio-diciembre del 2021 
Ainsi, il y a deux types de responsabilité de l'État, à savoir contractuelle et extracontractuelle, qui peuvent être pour faute ou sans faute. Dès lors, c'est dans cette large marge que l'on peut mettre en évidence des différentes expressions dans lesquelles le juge indemnise les personnes dont les espérances ou les attentes ont été trompées.

La réparation apparait comme un mécanisme de protection des personnes contre les dommages causés par les actions de l'administration ajustées ou contraires au droit. Dans cette mesure, trois éléments sont constitutifs de la responsabilité de l'État : le fait générateur, le préjudice et le lien de causalité (Truchet, 2019, p. 383). D’un côté, lorsqu’il s'agit de la responsabilité pour faute, le fait à l'origine du préjudice est représenté par tous les actes ou omissions fautifs et, en général, les « manquements qui pesaient sur l'auteur » (Truchet, 2019, p. 381). De l'autre côté, dans la responsabilité sans faute, le fait générateur est délimité à toutes les actions conformes au droit, c'est-à-dire qui ne sont pas fautives, mais qui causent un préjudice. Cependant, comme le préjudice ne résulte pas d'une défaillance du service, celui-ci ne peut être n’importe quel préjudice : il doit être anormal, c'est-à-dire grave et spécial (Dupuis et Guédon, 2004, p. 572). Il est là une spécificité du droit administratif permettant d'offrir des garanties et, dans certains cas, d'équilibrer les charges dans les relations entre les personnes publiques et les particuliers.

Le cas particulier analysé dans ce travail concerne les intérêts qui, sans être des droits acquis, peuvent trouver une protection à travers la responsabilité de l'État. Par ailleurs, il est nécessaire de noter que le dommage ou le préjudice pour être indemnisable doit remplir certaines conditions : il doit être personnel, direct et certain (Rougevin-Baville, 1992, p. 139).

La première caractéristique est le fait que le préjudice doive être personnel et direct, ce qui semble déterminer que la réparation du préjudice ne peut

Via Inveniendi Et Iudicandi

e-ISSN: 1909-0528 | DOI: https://doi.org/10.15332/19090528

Vol. 16 N.o 2 | julio-diciembre del 2021 
être réclamée que par le titulaire du droit ou pour celui qui a été pris en compte dans certains cas comme la victime du dommage, c'est-à-dire la personne directement concernée. Cette circonstance exclurait les tiers qui ne sont pas des victimes directes. Néanmoins, dans la jurisprudence française, non seulement la victime directe peut demander la réparation des dommages subis, mais aussi les personnes qui ont un intérêt juridiquement protégé (Dupuis et Guédon, 2004, p. 581), ce qui se reflète dans ce que l'on appelle les dommages subis par ricochet (également appelé « préjudice par ricochet ») ou préjudices réfléchis (ou « dommages réfléchis »), qui ne sont rien d'autre que des dommages indirects. Dès lors, le titulaire du droit ou la victime du préjudice subit un dommage direct et les tiers subissent un dommage indirect.

En plus, le dommage doit être certain, ce qui implique que le droit ou l'intérêt d'une personne a effectivement été affecté sans que cela n'empêche l'existence de dommages futurs, en les distinguant de ceux qui sont simplement éventuels. Cette distinction a été adoptée sur la base du degré d'incertitude qui, en cas du dommage futur, doit avoir un pourcentage très faible, ce qui détermine un certain degré de certitude. C'est une circonstance qui ne se présente pas dans le dommage éventuel, où l'incertitude est trop élevée pour être prise en compte pour donner lieu à une indemnisation.

En ce sens, la jurisprudence française se réfère au dommage futur comme étant la perte d'une opportunité claire de réaliser un droit ou d'atteindre un but, qui peut se refléter dans des intérêts économiques, de travail, de santé, entre autres, affectés par l'action publique ; compte tenu le degré considérable de certitude, on fait référence à la chance sérieuse.

De ces éléments généraux, on peut conclure que, quand bien même les intérêts tels que les attentes ou les espérances légitimes ne peuvent pas être considérées comme des droits rentrant dans le patrimoine d'un 
individu, cependant la réparation est possible. Il s'agit donc d'intérêts ou de droits futurs qui, selon la jurisprudence du Conseil d'État, peuvent être réparés lorsqu'il existe une forte certitude quant à leur survenance. C'est pourquoi « le fait que les actes de l'administration privent la victime de ces intérêts légitimement fondés peut donner lieu à une indemnisation ».

À cet égard, le préjudice futur, caractérisé par la perte d'une opportunité ou d'une chance sérieuse d'obtenir un droit ou d'atteindre un but ayant été poursuivi, peut être indemnisé (Salle, 1994) dans la mesure où il diminue ou élimine la probabilité de l'événement favorable qui devrait possiblement naître ou générer (Cour de Cassation, 6 juin 1990). Pour cette raison, et en raison de la forte probabilité de survenance, il peut être situé entre le préjudice certain et le préjudice éventuel, avec la caractéristique d'être indemnisable (Chartier, 2005, p. 615).

Cest bien la raison pour laquelle, dans la plupart des cas, lorsque les attentes légitimes sont trompées, le dommage produit ultérieurement sera indemnisé puisqu'une attente répond aux intérêts en cours de consolidation, de sorte que ce qui est mis en cause c'est la perte de la possibilité d'obtenir un bénéfice ou de consolider un droit dans le patrimoine d'une personne par l'action de l'État. C'est le cas, par exemple, lorsqu'une personne est illégalement interdite de participer à un concours administratif (Conseil d'État, Requête no 62342 du 1965).

Toutefois, il convient de préciser que, même si les espérances reposent principalement sur les dommages futurs, le juge administratif peut éventuellement ne pas reconnaître la perte de l'opportunité mais les frais engagés par la victime.

Une fois énoncées ces précisions sur les éléments constitutifs de la responsabilité, il convient de noter que dans le cas des attentes légitimes les dommages qui en résultent sont le plus souvent futurs, ce qui 
n'empêche pas qu'ils soient certains. C'est le cas, par exemple, dès que l'action de l'administration limite l'obtention d'un bénéfice ou la continuité de celui-ci, ce qui conduirait à une réparation. Dès lors, il faut déterminer quels événements et de quelle manière le juge administratif est parvenu à protéger les attentes légitimes car, comme l'affirme Silvia Calmes, il est évident que, à travers la théorie de la responsabilité de l'État pour faute et sans faute, il y a une logique de protection subjective de la confiance légitime (Calmes, 2001, p. 615).

D’une part, dans le cas de la responsabilité pour faute, le critère des attentes ou des espérances légitimes peut être mis en évidence dans les événements des promesses et des assurances que l'administration fait aux administrés mais qui, par la suite, ne sont pas remplies. Une action qui fait naitre chez les destinataires un sentiment de confiance, une espérance que ce qui a été promis ou assuré sera réalisé, comme l'édition d'un acte administratif ou la conclusion d'un contrat.

Les promesses ou assurances, même si elles n'ont pas de valeur juridique (Yannakopoulos, 1997, p. 535), en raison de leur influence sur le destinataire, permet au juge de reconnaître aux victimes de bonne foi le droit à une indemnisation selon les termes de la responsabilité de la puissance publique pour faute (Calmes, 2001, p. 620). En effet, si l'administration donne confiance à l'administré, par ses actions, en l'existence d'une situation juridique, et puis contrairement aux attentes, elle se désintéresse des conséquences qui découlent de sa promesse, le juge considère qu'il peut s'agir là d'une faute, surtout lorsque « l'administration a incité la victime à « engager des frais » (Conseil d’État, Requête $\mathrm{n}^{\mathrm{o}}$ 87909 du 1973; Plessix, 2016, p. 799). 
On peut songer aux attentes qui peuvent être générées dans l'édition d'actes unilatéraux tels qu'un permis de construire ${ }^{1}$, lorsque l'autorité compétente a fait croire qu'il serait accordé. Alors, si les personnes publiques agissent contrairement à la parole donnée, le destinataire peut demander la réparation des dommages par la faute commise.

Cependant, il est évident que, pour que le juge reconnaisse les préjudices dérivés des promesses non tenues, des assurances ou promesses trompeuses, au-delà du fait d'examiner la faute de la collectivité publique, il doit prendre en compte les dommages causés en raison de la méconnaissance de l'espérance ou la croyance/confiance que l'administration avait créé chez l'administré, ainsi que l'affirme Benoît Plessix :

[...] en sanctionnant les promesses abusives d'un pouvoir public en qui l'on fait confiance, en se plaçant du point de vue des intéressés et en tenant compte de leur ressenti, le juge administratif français fait de nouveau usage de l'idée de confiance légitime, ce qu'avait d'ailleurs pressenti Maurice Hauriou. Une fois de plus en effet, tout ce régime de responsabilité extracontractuelle pour faute ne se comprend qu'au regard de ses attentes déraisonnablement déjouées par l'Administration (Plessix, 2016, p. 799).

De ce fait, même s'il s'agit là d'une forme classique d'attribution de la responsabilité extracontractuelle pour faute, celle-ci résulte de la méconnaissance de cet intérêt qui s'est créé chez la personne concernée par le changement d'action de la collectivité publique. Dès lors, bien qu'il ne soit pas possible de parler d'un droit, dans la mesure où il n'existe pas

\footnotetext{
${ }^{1}$ Conseil d'État (1973, Requête 87909), dans lequel le haute juridiction reconnait la réparation d'un préjudice en raison des espérances trompés dans les termes suivants « cons. qu'en poursuivant le recouvrement de participations financières au titre d'un permis de construire non encore délivré, et en laissant croire ainsi à la société que ledit permis lui était acquis, les services de la ville de Nice ont commis une faute qui a causé à la société un dommage; que ce dommage correspond, à l'exclusion de tout manque à gagner, au montant des dépenses qu'elle a inutilement exposées, au titre du projet de construction ».
}

Via Inveniendi Et Iudicandi

e-ISSN: 1909-0528 | DOI: https://doi.org/10.15332/19090528

Vol. 16 N.0 2 | julio-diciembre del 2021 
d'acte juridique l'imposant explicitement, comme peut être un contrat ou un acte administratif, le fondement de l'intérêt qui a été créé permet que les attentes trompées soient réparées.

D’ailleurs, même si le juge ait souvent été réticent à l'idée de reconnaitre ces figures, de sorte que, dans certains évènements, « il ne conclut pas à la responsabilité à défaut d'engagement formel » (Conseil d'État, 8 février 1952, cité par Calmes, 2001, p. 621) ou exclut les cas dans lesquels le requérant a été imprudent (Conseil d'État, 24 avril 1964), c'est là une expression claire de la protection des espérances ou des attentes légitimes, dans laquelle le juge reconnaît les préjudices causés par la violation des intérêts des particuliers visant à ce que l'acte juridique soit édicté.

C'est un exemple qui peut être mis en évidence de la même manière dans le domaine contractuel, comme l'affirme Benoît Plessix quand, au stade des négociations, c'est-à-dire pendant l'étape précontractuelle, l'administration génère chez l'opérateur économique une attente ou une espérance que le contrat sera conclu (Plessix, 2016, p. 799). C'est le cas de l'affaire du 6 décembre 2017 dans lequel le Conseil d'État a engagé la responsabilité pour faute d'une collectivité territoriale « au motif que celui-ci a fortement incité la société requérante à exécuter certaines prestations et a promis formellement à celle-ci la signature d'un contrat » (Conseil d'État, Requête no 400406 du 2017).

La collectivité avait retenu l'offre de la société requérante pour réaliser un projet en vue de construire un ensemble immobilier, mais la personne publique s'est ensuite désengagée du processus de passation. Cependant, compte tenu du fait que la société avait exécuté des actes avec la conviction que le contrat serait conclu, la réparation des dommages causés a été demandée, une prétention accueillie par le Conseil d'État qui a établi que la collectivité était responsable de réparer les préjudices en raison de la promesse fautive. 
Bien que le contrat n'était conclu, la collectivité avait incité la société requérante à exécuter des actions telles que la réalisation des études préalables et le dépôt des demandes d'autorisation d'urbanisme, avec la ferme conviction que le contrat allait être célébré. C'est la raison pour laquelle la collectivité a été condamnée à indemniser au requérant les divers frais qu'elle a engagés, mais il a rejeté le préjudice subi sur le manque à gagner, en raison du fait que l'acceptation d'une offre ne créé pas le droit de conclure le contrat et, donc, celui-ci ne pouvait pas être reconnu.

D'autre part, dans le cas de la responsabilité sans faute, c'est-à-dire des préjudices qui résultent des actions légitimes de l'administration mais aussi du législateur, ceux-ci prennent une importance particulière concernant la protection des attentes légitimes déçues, dans la mesure où même si une action est conforme au droit, elle peut engendrer des dommages qu'une personne ne devrait pas supporter.

Silvia Calmes note également que la responsabilité sans faute générée par la rupture de l'égalité devant les charges publiques « contient potentiellement les éléments nécessaires pour protéger la confiance légitime des particuliers et qui peuvent être notamment utiles lorsqu'aucun abus pouvant être qualifié de faute n'a été commis par la personne publique en cause » (Calmes, 2001, p. 629).

Sur ce point, il est nécessaire de préciser comment, à travers la responsabilité de l'État pour la rupture de l'égalité devant les charges publiques, soit par le fait de l'administration, soit par le fait du législateur, les espérances légitimes sont protégées. Étant donné qu'elles prennent une importance particulière en tant que critère pour établir la responsabilité de l'État tel que le précise Camille Broyelle, qui établit que les attentes légitimes représentent un critère qui a été présent tout au long des considérations du juge administratif, en partant de la responsabilité 
contractuelle où la confiance légitime dans la théorie du fait du prince joue un rôle considérable pour déterminer s'il y a une obligation de réparation, notamment lorsque l'État bouleverse les conditions du contrat et, en général, l'état du droit du contrat. Ceci peut engendrer, chez le contractant, des préjudices résultant de la méconnaissance de l'intérêt de confiance ou d'espérance que les conditions contractuelles envisagées seraient maintenues, mais, au-delà du contrat, que l'état de droit applicable dans la relation contractuelle resterait inchangé (Broyelle, 2018).

C'est une position qui peut être applicable à la responsabilité extracontractuelle pour la rupture de l'égalité devant les charges publiques depuis l'arrêt Couitéas en 1923 dans les événements où l'administration, avec ses omissions, est celle qui a généré les préjudices et à partir de l'arrêt la Fleurette en 1938 où la responsabilité du législateur est admise en raison de ses actes (Broyelle, 2009, p. 321).

En effet, l'arrêt Couitéas (Conseil d'État, Requête no 3828448688 du 1923), bien que d'aucuns n'y voient pas une révolution juridique (Bonichot, 1999, cité par Dupuis et Guédon, 2004, p. 576), car la forme qu'il donne à la responsabilité avait déjà été précisée dans des arrêts antérieurs (Deguergue, 1994), acquiert néanmoins une pertinence particulière en raison des critères sur lesquels se fonde pour arriver à la réparation des dommages causés par une action conforme au droit. En l'occurrence, le requérant demandait la réparation du préjudice causé pour la non-exécution d'une décision du tribunal civil de Sousse, d'après laquelle devait faire expulser les indigènes d'un terrain dont Monsieur Couitéas était le propriétaire. Or, même si la décision d'abstention de l'administration fût légitime, étant donné que celle-ci était fondée sur la protection de l'ordre public et la garantie de l'intérêt général, ce qui permettait de méconnaître l'intérêt du requérant, une décision qui s'étend à d'autres cas comme les jugements ordonnant l'expulsion d'occupants 
sans titre de logement (Conseil d'État, Requête $n^{0} 20648$ du 1983), le Conseil d'État a établi que la victime avait manifestement un droit de réparation pour l'action sans faute de l'administration en raison du principe d'égalité devant les charges publiques.

Ainsi, si une action régulière crée des préjudices qu'une personne ne devrait pas supporter, il faut les réparer mais, comme le précise Camille Broyelle, ce n'était pas le seul critère pris en compte pour engager la responsabilité de l'État, car celle-ci résulte également de la méconnaissance des espérances ou des attentes qui sont nées chez le requérant que la décision juridictionnelle serait exécutée et que l'administration agirait conformément à cette décision.

Camille Broyelle signale que

[...] dans l'arrêt Couitéas, un autre élément a joué, et dont le Conseil d'État rend compte dans la première partie de son raisonnement: «Considérant qu'en prenant, pour les motifs et dans les circonstances rappelées ci-dessus, la décision dont se plaint le sieur Couitéas, ledit gouvernement n'a fait qu'user des pouvoirs qui lui sont conférés en vue du maintien de l'ordre et de la sécurité publique dans un pays de protectorat; Mais, considérant que le justiciable, nanti d'une sentence judiciaire dûment revêtue de la formule exécutoire, est en droit de compter sur l'appui de la force publique pour assurer l'exécution du titre qui lui a été ainsi délivr».

Basilio Couitéas pouvait compter sur l'appui de la force publique parce que la décision de justice pouvait lui donner légitimement ces espérances. Une espérance a été trahi, une prévision déjouée. Tel est l'élément déterminant selon nous, qui ouvre droit à réparation dans l'affaire Couitéas. À ce stade, ce que l'on appellera plus tard la « théorie du fait du prince » a joué un rôle majeur ». (Broyelle, 2009, p. 321)

Via Inveniendi Et Iudicandi e-ISSN: 1909-0528 | DOI: https://doi.org/10.15332/19090528 
En effet, même s'il s'agit, dans ce procès, de préjudices causés à un droit qui a été explicitement reconnu par une décision de justice, l’importance réside dans l'intérêt qui résulte des effets de la décision qui a accordé un tel droit. Ainsi, ce que Monsieur Couitéas espérait c'était l'adoption de mesures nécessaires par l'administration pour expulser les indigènes qui occupaient sa propriété, c'est-à-dire que les effets attendus de la décision judiciaire soient respectés.

Ces circonstances ont ensuite été étendues aux actes généraux et impersonnels, tels que les lois ou les actes réglementaires, car, si l'administration et le législateur sont libres de modifier leurs dispositions sans que celles-ci soient contraires au droit, la responsabilité de l'État pour la rupture de l'égalité devant les charges publiques peut être une limite où les intérêts des destinataires doivent être pris en compte et où les espérances ou les attentes jouent également un rôle considérable.

Dès lors, il faut analyser l'arrêt La Fleurette de 1938 (Conseil d'État, Requête $\mathrm{n}^{0} 51704$ du 1938), qui est le précurseur de la responsabilité du fait des lois dans la mesure où il a admis «l'ouverture d'un droit à indemnité (ou indemnisation) en réparation des conséquences dommageables d'une loi» (Dupuis et Guédon, 2004, p. 566), sans oublier que ce type de responsabilité s'applique non seulement aux lois, mais aussi aux autres actes ayant une portée générale comme les actes réglementaires (voir Conseil d'État, 22 février 1963 ; Conseil d'État, 13 mai 1987) et les traités internationaux (voir Conseil d'État, 30 mars 1966 ; Conseil d'État, 29 octobre 1976 ; Conseil d'État, 29 décembre 2004).

Dans le cas de l'arrêt La Fleurette, il s'agissait d'une entreprise produisant de la crème, une activité qui était exercée selon les autorisations légales que l'État lui avait octroyées. Toutefois, par la suite, une loi visant à protéger le marché du lait a interdit la fabrication de produits remplaçant la crème naturelle, ce qui a manifestement nui à l'activité de la société 
requérante. Cependant, le Conseil d'État a imposé des conditions très restrictives pour reconnaître la réparation du préjudice, telles que :

Que l'activité touché ne soit pas nuisible pour la société, que l'intention du législateur n'ait pas été d'exclure toute indemnisation ou qu'il n'ait pas lui-même ordonné cette dernière et que le nombre de victimes d'une loi voté nécessairement dans l'intérêt général soit extrêmement réduit et leur préjudice particulièrement grave (Truchet, 2019, p. 413).

Depuis lors, on limite les cas dans lesquels la responsabilité de l'État est engagée du fait du législateur. Mais en tout état de cause on a reconnu que les victimes dont les dommages résultent de l'imprévisibilité de la loi peuvent être indemnisées, sur la base de la rupture de l'égalité des charges publiques, c'est-à-dire que le préjudice causé par la loi devait être d'une ampleur telle qu'une personne ne devrait pas supporter.

Toutefois, il est possible d'appliquer les critères respectifs de la confiance légitime pour établir s'il existe une obligation de réparation à la victime dans ces actes à caractère général, comme l'affirme Camille Broyelle :

L'arrêt La Fleurette de 1938 peut en effet être compris en ce sens. On peut considérer que si le Conseil d'État répare le préjudice causé à la société La Fleurette par l’interdiction (énoncée dans la loi du 29 juin 1934) de fabriquer une crème laitière ne provenant pas exclusivement du lait, c'est en raison d'une confiance légitime trompée. La société La Fleurette comptait sur le maintien de « l'état de choses existant »; sa confiance, légitime, a été trompée; il y a lieu à réparation. (Broyelle, 2009, p. 321)

Ceci le rend d'autant plus pertinent en ce qui concerne la protection des attentes ou de la confiance légitime que, dans le cas de l'arrêt Couitéas, dans la mesure ou dans l'affaire Couitéas, il s'agissait d'un intérêt qui découlait d'un droit spécifique qui avait été affecté par les actions de

Via Inveniendi Et Iudicandi

e-ISSN: 1909-0528 | DOI: https://doi.org/10.15332/19090528

Vol. 16 N.o 2 | julio-diciembre del 2021 
l'administration tandis que, dans le cas de l'affaire La Fleurette, il apparait que l'intérêt va bien plus loin car, si en principe la société n'avait pas un droit consolidé, elle avait une espérance ou une attente qu'elle pourrait continuer à exercer son activité conformément à la réglementation antérieure. En ce sens, lorsque la nouvelle disposition supprime la faculté d'exercer son activité économique, ses espérances sont déçues et c'est dans ce contexte que l'on pourrait interpréter la nécessité de réparer le préjudice causé à la société requérante.

Cependant, le juge administratif a longtemps été réticent à reconnaître la responsabilité du fait des lois, de sorte que celle-ci était écartée dans la plupart des cas lorsque le législateur gardait le silence face à la reconnaissance d'une indemnisation, comme c'était le cas pour les événements d'activités frauduleuses (Conseil d'État, 1 mars 1940, p. 8), et lorsqu'il existait des motifs d'intérêt supérieur justifiant l'adoption de la loi (Conseil d'État, 24 avril 1953, p. 191). Mais la ligne de pensée de l'arrêt La Fleurette a été reprise dans des arrêts ultérieurs comme l'affaire Coopérative Ax'ion en 2005, dans lequel le juge administratif a reconnu la responsabilité de l'État du fait des lois à une société qui avait été affectée par une décision conforme à une nouvelle loi. Néanmoins, dans ce cas, le Conseil d'État a apporté quelques précisions pour déterminer une obligation de réparation : le silence du législateur doit alors être compris comme ouvrant droit à indemnisation. Dans le même contexte, pour que le dommage soit réparable, il doit être anormal et spécial, en tenant compte du fait que l'intérêt général ne peut être compris comme un élément excluant toute indemnisation. En somme, il est établi qu'une personne est fondée à réclamer l'indemnisation du préjudice qu'elle a subi lorsque « excède les aléas que comporte nécessairement une telle exploitation » (Conseil d'État, Requête no 266564 du 2005). 
Cette dernière condition permet de démontrer que le préjudice se forme lorsque la victime ne pouvait pas prévoir son apparition découlant de l'action du législateur, tel que l'établit Camille Broyelle (2009, p. 321). Dans ce contexte, pour déterminer si une personne a droit à la réparation des dommages causés, le juge ne se limite pas à établir si l'action du législateur a engendré des dommages tendant à rompre l'égalité devant les charges publiques. Il doit aussi examiner si l'intérêt de confiance ou l'espérance que la victime avait déposée a été trompé avec l'action du législateur (Broyelle, 2009, p. 321).

C'est une évolution de la jurisprudence qui permet de mettre en évidence que, de plus en plus, les espérances légitimes deviennent un critère pour déterminer s'il y a lieu à une responsabilité de l'État pour la rupture de l'égalité dans les charges publiques, ainsi que le souligne Camille Broyelle :

C'est bien ici la confiance légitime trompée qui ouvre droit à réparation. La précision selon laquelle le préjudice était grave et spécial apparait, dans cette affaire comme dans toutes celles jugées après l'arrêt Ax'ion, comme un simple hommage à l'égalité devant les charges publiques.

(Broyelle, 2009, p. 321)

Toutes ces expressions permettent d'affirmer que, même si le droit français n'établit pas expressément un principe transversal comme c'est le cas dans le système juridique colombien, tel que la confiance légitime, il existe une série de mécanismes permettant d'offrir des garanties aux intérêts particuliers qui ne sont pas considérés comme des droits acquis. De plus, il en est de même dans le domaine de la responsabilité de l'État, où sont présentés différents exemples dans lesquels le fondement de la réparation des préjudices est la protection des intérêts qui peuvent être appelés attentes ou espérances légitimes, surtout dans le domaine de la responsabilité sans faute. 
Il est cependant intéressant d'évaluer la direction que prendra le Conseil d'État en termes de responsabilité, lorsqu'il s'agit de lois contraires aux traités internationaux et à la Constitution, compte tenu du fait que les attentes et les espérances, entre autres, sont des notions reconnues par la Constitution et par la Convention européenne des droits de l'homme, étant donné que la possibilité d'engager la responsabilité de l'État par le fait des lois inconstitutionnelles ou contraires aux engagements internationaux a été récemment admise. Cela était, auparavant, impensable, de sorte que tout préjugé par le fait du législateur était transféré dans le domaine de la responsabilité sans faute. On ne peut pas fermer la porte à l'introduction de nouvelles mesures, afin d'offrir des garanties aux particuliers dans leurs relations avec l'État, mais on ne peut pas non plus affirmer que le droit administratif français est totalement objectif et qu'il rejette toute manifestation de la subjectivité parce que, comme cela a été démontré, il existe des mécanismes similaires à ceux qui sont adoptés dans des systèmes juridiques ayant une forte composante subjective. Il est donc nécessaire d'établir les formes de protection prévues par le droit colombien.

\section{La réparation des préjudices : une forme essentielle de protection des espérances légitimes en Colombie}

La protection de l'espérance légitime générée par l'action de l'État Colombien s'inscrit dans le cadre de la reconnaissance des droits futurs ou des mesures visant à les consolider. Néanmoins, lorsque les espérances légitimes ont été trompées, la réparation des préjudices devient l'un des mécanismes de protection les plus fréquents et les plus efficaces, compte tenu du fait que l'ordre juridique colombien a reconnu comme titre d'imputation de la responsabilité de l'État, la violation des attentes ou des

Via Inveniendi Et Iudicandi

e-ISSN: 1909-0528 | DOI: https://doi.org/10.15332/19090528

Vol. 16 N.o 2 | julio-diciembre del 2021 
espérances légitimes sous le principe de la confiance légitime (Vargas, 2018).

Il convient à cet égard de procéder à une analyse des mécanismes énoncés :

En effet, dans un premier temps, le mécanisme idéal de protection serait la reconnaissance des droits qui sont en cours de consolidation et pour lesquels cette espérance légitime a été créé puisque, finalement, ce que l'on recherche avec ce type d'intérêts fondés sur des bases objectives, c'est sa configuration ou sa matérialisation. Dans le même contexte, l'adoption des mesures nécessaires tendant à leur consolidation ou à leur adaptation à la nouvelle situation est optimale pour protéger les intérêts individuels.

Ces deux formes sont présentes en droit colombien dans divers domaines. C'est par exemple le cas dans les opérations complexes telles que les concours de la fonction publique, en ce qui concerne les participants qui veulent occuper un poste dans la fonction publique. Dans ce cas, bien que le droit ne soit consolidé que par l'acte administratif de nomination, lorsqu'un candidat est retenu dans la procédure de sélection, il attend sa nomination s'il remplit tous les critères et toutes les conditions prévus tant par la loi que par l'acte d'ouverture de la opération. De ce fait, si l'autorité publique décide de nommer un autre candidat qui ne remplit pas les conditions énoncées, le candidat qui espérait être nommé voit trompées ses espérances légitimes par l'action de l'État, dans la mesure où, même s'il n'était pas titulaire du droit, il avait une croyance forte que celui-ci serait défini.

Dans ce cas, le Conseil d'État a reconnu l'existence du droit subjectif futur susceptible d'être garanti en l'accordant à l'individu à qui l'espérance légitime d'être nommé a été méconnue (Conseil d'État Colombien, Arrêt du 17 mai 2012). C'est une circonstance qui a des effets par le biais du 
recours contentieux administratif correspondant, en vertu duquel l'annulation de l'acte ordonnant la nomination d'une personne autre que celle qui aurait dû être désignée est demandée, mais en outre le rétablissement en raison de la violation de l'Espérance ou de l'attente fondée, ordonnant ainsi la nomination de la personne qui devait occuper le poste selon les dispositions préétablies.

Dans le cas précédent, il s'agit de matérialiser le droit subjectif futur, puisque la décision prise par la juridiction administrative est de reconnaître l'Espérance ou l'attente légitime et d'accorder le droit virtuel ; c'est-à-dire rendre effectif le but poursuivi.

Une autre expression analogue est celle de la méconnaissance des attentes légitimes en matière fiscale, un fait qui présente une similitude particulière avec le droit français où c'est dans ce domaine spécifique que les espérances légitimes ont été expressément reconnues comme étant des droits. En Colombie, lorsqu'une norme confère un avantage ou un bénéfice fiscal, une espérance légitime peut être générée chez le contribuable, en tenant compte d'exigences telles que

[...] la validité de la norme ; son existence obligatoire, qui n'a pas fait l'objet de modifications, ce qui a généré des effets prévisibles significatifs, c'est-à-dire qu'elle a conduit les particuliers de bonne foi à adapter leur comportement selon ce qu'elle prescrit. (Hernández, 2007, p. 457)

Toutefois, si l'avantage convenu est ultérieurement supprimé ou modifié en limitant les destinataires, en respectant tous les paramètres légaux, l'attente du contribuable de continuer à être titulaire de cet avantage fiscal sera méconnue. En l'occurrence, la Cour Constitutionnelle colombienne a voisé que :

l'État doit donner à la personne concernée le temps et les moyens de s'adapter à la nouvelle situation, par exemple en prévoyant une période

Via Inveniendi Et Iudicandi

e-ISSN: 1909-0528 | DOI: https://doi.org/10.15332/19090528

Vol. 16 N.o 2 | julio-diciembre del 2021 
de transition ; mais, dans certaines situations, la protection des attentes légitimes peut exiger que l'avantage fiscal ne soit pas abrogé o modifié pendant la période durant laquelle s'applique le terme pour que les contribuables en bénéficient.

À cet égard, bien que la règle soit l'adoption de mesures transitoires pour que le destinataire s'adapte à la nouvelle règle, dans certains cas, le droit futur peut être reconnu. Cela signifie qu'il est possible de donner l'avantage fiscal attendu, dans la mesure où, les dispositions favorables seront maintenues, normalement, lorsque les bénéfices sont prévus par la loi pour une durée précise (Hernández, 2007, p. 457).

De même, une autre manifestation de la protection des espérances légitimes par le maintien des mesures favorables, et par conséquence la reconnaissance des droits futurs, se trouve dans le domaine des pensions, dans ce cas précis lorsqu'une norme ultérieure établit des exigences plus strictes que la norme précédente. Le juge constitutionnel a exigé l'adoption de mesures temporaires tendant à maintenir le régime antérieur pour ceux qui sont sur le point de prendre leur retraite et remplissent toutes les conditions requises pour qu'ils puissent consolider le droit, c'est-à-dire obtenir leur pension de retraite.

Dans cette mesure, la Cour Constitutionnelle a établi que le législateur pouvait modifier les conditions qui régissaient le droit lorsqu'il s'agit de simples attentes et qu'au contraire, lorsqu'il s'agit de droits acquis, il existait une interdiction pour le législateur d'adopter des actes ayant des effets rétroactifs, c'est-à-dire qu'ils ne peuvent pas être méconnus ou modifiés. Cependant, dans le cas des attentes légitimes qui sont considérées comme un point intermédiaire entre les deux expressions mentionnées, puisque l'individu se trouvait aux portes de consolider ses aspirations. Dans ce cas, celui d'obtenir la pension de retraite, elles doivent être l'objet de la considération protectrice de la part du législateur et ne 
peuvent être modifiées de manière arbitraire (Cour Constitutionnelle Colombienne, Décision C-428 de 2009).

Ainsi, la Cour a souligné qu'il fallait maintenir les conditions qui régissaient le droit afin d'en assurer sa consolidation et, à cet égard, d'assurer la formation de l'espérance légitime (Cour Constitutionnelle Colombienne, Décision T-662 de 2011).

Pourtant, en tout état de cause, l'intérêt général joue un rôle très important dans l'activité de l'État, de sorte qu'il y ait des occasions où les mesures qui accordent le droit ne peuvent être prises et, au lieu de cela, il faut réparer les dommages causés à travers les indemnisations financières, en adoptant la forme de protection la plus fréquente des attentes légitimes. Cette approche se concrétise par la responsabilité de l'État qui, en Colombie, se fonde sur l'article 90 de la Constitution, qui prévoit l'obligation de réparer les dommages causés par l'action ou l'omission des autorités publiques. En ce sens, la norme constitutionnelle a reconnu les trois éléments traditionnels de la responsabilité de l'État qui se traduisent par un préjudice indemnisable (Conseil d'État Colombien, Arrêt du 23 mai 2012 ; Conseil d'État Colombien, Arrêt du 28 janvier 2015), une action ou omission de l'administration, et un lien de causalité entre le comportement et le dommage (Conseil d'État Colombien, Arrêt du 15 novembre 2011). Il convient d'indiquer que la responsabilité de l'État en Colombie peut être extracontractuelle dans la mesure où celle-ci résulte d'actions autres que les contrats. De plus, la responsabilité de l'État peut également être contractuelle dans les cas qui découlent d'un contrat. Par ailleurs, la responsabilité dans la procédure de passation de marché peut être précontractuelle ou post-contractuelle.

De même, le système juridique colombien a adopté des titres ou des formes d'attribution de responsabilité à l'État qui vont de la responsabilité 
pour faute, lorsque celui-ci agit irrégulièrement, c'est-à-dire éloignée du bon fonctionnement du service public (Conseil d'État Colombien, Arrêt du 24 janvier 2019), à la responsabilité sans faute ou objective qui, en ce qui concerne ce travail, implique celle dite de dommage spécial (Conseil d'État Colombien, Arrêt du 14 mars 2019) qui se forme face à la rupture de l'équilibre devant les charges publiques.

Dans les circonstances qui précèdent et avec les précisions qui s’imposent sur les formes d'attribution de la responsabilité de l'État, il convient d'indiquer que lorsque l'État, par l'intermédiaire d'une autorité publique, méconnait une espérance légitime et cause un préjudice qu'une personne ne devrait pas supporter, il est tenu d'indemniser le dommage qu'il a causé, soit à la suite d'un comportement irrégulier, c'est-à-dire d'une faute du service, soit à la suite d'une action qui, étant légitime, entraîne un préjudice anormal et spécial. Ceci donne lieu à une responsabilité objective dans l'expression du dommage spécial ou par la rupture devant les charges publiques. De plus, le Conseil d'État, dans une nouvelle approche qui a été novatrice dans le système juridique colombien, a appelé la responsabilité pour la violation des attentes légitimes comme conséquence du principe de la confiance légitime.

En effet, il arrive que l'État, par l'exercice de ses fonctions, porte atteinte aux espérances ou attentes légitimes, c'est-à-dire aux intérêts des individus qui possèdent d'une certaine façon une valeur juridique. Dans ce cas, il a une obligation d’indemniser le préjudice causé, lorsque celui-ci est spécial et anormal, c'est-à-dire qu'il s'adresse à un nombre spécifique de personnes qui ne devraient pas supporter les dommages subis.

En ces termes, le Conseil d'État a traité les espérances légitimes trompées dans le champ de la responsabilité de l'État de la manière suivante :

Via Inveniendi Et Iudicandi

e-ISSN: 1909-0528 | DOI: https://doi.org/10.15332/19090528

Vol. 16 N.० 2 | julio-diciembre del 2021 
Il convient de noter qu'en l'espèce : (i) les parties ont légitimement exercé leur droit constitutionnel d'accès à l'administration de la justice en engageant une action en réparation directe et en faisant appel de l'ordonnance par laquelle leur demande a été initialement rejetée. (ii) l'administration a développé un comportement positif et juridiquement pertinent, ce qui a généré une attente légitime sur le comportement futur, dans la mesure où, le tribunal administratif a déterminé qu’il n'avait pas lieu de déclarer la résiliation du contrat et, par conséquent, il fallait continuer le procès, afin de rendre une décision de fond et (iii) par la suite, le juge a exercé un comportement intempestif, qui a porté atteinte à l'attente générée chez les requérants. (Conseil d'État Colombien, Arrêt du 31 mars 2016)

Tel qu'il ressort de l'arrêt précité, l'administration publique peut susciter des attentes ou des espérances avec ses actes objectifs, de sorte que cellesci constituent une expression extérieure d'un comportement futur uniforme et constant qui, en règle générale, se matérialise par l'acquisition très probable d'un droit subjectif par un individu. Néanmoins, de la même manière, il est possible que les autorités publiques, de façon imprévue ou intempestive, méconnaissent l'espérance ou les attentes qu'elles ont suscitées, ce qui fait que le droit futur fondé sur le comportement antérieur de l'administration soit considère trompé.

La protection de l'espérance légitime, dans ce cas, n’implique pas que le droit entre dans le patrimoine de la personne, sinon nous serions face à un droit acquis. Toutefois, il y a une forte probabilité qu'elle entre dans le patrimoine à l'avenir ou simplement que celle-ci soit consolidée. C'est la raison pour laquelle il s'agit d'un droit futur ou en cours de consolidation et l'Administration, par son comportement intempestif, fait obstacle à l'acquisition de ce droit. C'est ce qu'une doctrine a appelé un intérêt particulier ou un avantage personnel. 
Dans un premier temps, la jurisprudence du Conseil d'État avait établi trois conditions pour qu'une espérance soit considérée comme ayant été trompée et qu'une obligation de réparation soit créée. Ces conditions sont :

I. l'existence d'une perte de chance, II. L'impossibilité définitive d'obtenir le profit ou d'éviter le préjudice ; et III. la victime doit se trouver dans une situation susceptible de lui permettre d'obtenir le résultat escompté; c'est-à-dire qu'il faut analyser si la personne concernée se trouvait dans des conditions factuelles et juridiques propres à lui permettre d'obtenir le profit pour lequel elle préconisait ou d'éviter le mal qu'elle cherchait à éviter. (Conseil d’État Colombien, Arrêt du 11 août 2010)

Dès lors, ces exigences impliquent :

i) la perte de possibilité de réalisation d'un évènement favorable, c'est-àdire que, bien qu'il s'agisse d'une espérance ou d'une attente, une occasion sérieuse, crédible se présente pour obtenir un droit ou un avantage, ce qui permet une grande probabilité d'atteindre le but espéré, mais qui est perdue par l'action de l'État. Lors de ce premier cas, on est confronté à un concept développé à partir du droit français, dans la mesure où la condition que le dommage soit certain n'empêche pas qu'il s'agisse d'une espérance ou simplement d'un intérêt à obtenir un avantage dans le futur. C'est un préjudice qui découle de la perte effective ou certaine de la possibilité de réalisation d'un événement désiré.

ii) L'impossibilité d'obtenir le profit ou l'avantage recherché et généré, ce qui montre une réalité négative ; c'est-à-dire la certitude que le droit ou le bénéfice ne sera pas atteint en raison de la méconnaissance de l'espérance ou de l'attente légitime.

iii) Enfin, la troisième condition posée fait référence à la légitimité de l'attente et du droit, de l'intérêt ou de l'avantage recherché, ce qui détermine les postulats de validité ainsi que l'aptitude à atteindre le but 
proposé ; qui se matérialise par l'existence de conditions factuelles et juridiques à la tête de l'individu pour réaliser l'intérêt recherché. Le Conseil d'État a concrétisé les critères en question de la manière suivante :

Cette position jurisprudentielle au sein de la salle du contentieux administratif du Conseil d'État précise que la perte d'opportunité ou de chance est un dommage en soi ayant une identité et des caractéristiques propres... Le fondement de la perte d'opportunité en tant que dommage autonome est constitué de deux éléments, l'un de certitude et l'autre d'incertitude. Le premier concerne l'existence de l'attente, celle-ci devant être vraie et raisonnable, comme en ce qui concerne la privation de celleci, car si le fait dommageable infligé par le tiers n'était pas intervenu, la victime aurait gardé intact l'Espérance d'obtenir à l'avenir un gain ou d'éviter un préjudice. En ce qui concerne les réclamations pour perte de chance, la Chambre précise qu'elles peuvent être présentées de deux manières, l'une positive -chance de gain- et l'autre négative -chance d'éviter une perte-. Elle est positive lorsque la victime s'attend légitimement à recevoir un avantage ou à acquérir un droit, mais que la conduite d'un tiers empêche définitivement l'espérance de concrétisation. Elle est négative lorsque la victime est plongée dans une démarche causale défavorable et qu'elle s'attend à ce que l'intervention d'un tiers évite ou élude un préjudice, mais qu'en raison de l'omission ou de l'intervention défectueuse de ce tiers, le résultat dommageable se produit et la victime subit le préjudice indésirable. (Conseil d'État Colombien, Arrêt du 5 avril 2017)

Dans les circonstances qui précèdent et dans le cas où les espérances légitimes étaient trompées par la cause d'un préjudice indemnisable du fait de la perte de la possibilité d'acquérir un droit futur ou un avantage, la responsabilité de l'État pour faute serait engagée si l'action qui gênait le préjudice était illégale ou contraire au droit et sans faute dans le cas où le fait entrainant le dommage était légale ou conforme au droit. Cependant,

Via Inveniendi Et Iudicandi

e-ISSN: 1909-0528 | DOI: https://doi.org/10.15332/19090528

Vol. 16 N.० 2 | julio-diciembre del 2021 
à partir de 2015, le Conseil d'État a créé une nouvelle forme d'attribution de responsabilité de l'État indépendant pour violation des espérances légitimes sur la base du principe de la confiance légitime, à travers l'analyse de l'activité de l'État-législateur.

En effet, la jurisprudence colombienne a précisé que l'État-législateur pouvait créer des états de confiance et des attentes ou des espérances légitimes, donnant lieu à des situations juridiquement protégées. Dès lors, lorsque celles-ci sont trompées, il s'agit d'une circonstance qui entraine l'existence des raisons ou des motifs d'imputation ou d'attribution de la responsabilité de l'État.

Toutefois, selon la nouvelle position du Conseil d'État, pour qu'une attente légitime génère une obligation de réparation, les conditions suivantes doivent être remplies :

i) l'existence d'une disposition de l'État à l'égard de laquelle des attentes légitimes sont nées ; ii) l'existence d'un comportement homogène et constant de l'État, qui conduit à l'établissement d'attentes légitimes ; iii) l'individu accomplit des actes qui affectent son domaine patrimonial ou extrapatrimonial ; iv) l'action imprévisible et intempestive engendrant la violation aux attentes ou aux espérances légitimes et v) la méconnaissance des obligations d'adaptation ou d'adéquation. (Conseil d'État Colombien, Arrêt du 31 août 2015)

Les exigences ci-dessus permettent de montrer que la protection d'une espérance dans le domaine de la responsabilité de l'État n'est pas absolue, mais qu'elle doit remplir les conditions énoncées.

Premièrement, il doit y avoir l'existence d'une disposition de l'État correspondant au fondement objectif de l'espérance. Deuxièmement, la configuration de cette espérance ou de cette attente, qui découle de l'action continue, doit être répété et ininterrompu par l'État. Troisièmement, il 
faut aussi des préjudices patrimoniaux ou extra patrimoniaux, étant donné que l'individu peut, en raison de ses espérances fondées, réaliser des actions qui produisent un impact normalement économique, comme peut être le cas d'investissements fondés sur le bénéfice qui sera réalisé à l'avenir, c'est alors à partir de ces affectations que sera déterminé le montant à indemniser. Quatrièmement, il est nécessaire de compter sur l'existence d'une action inattendue de l'autorité publique altérant les conditions d'action continue et homogène qu'elle développait et qui a conduit à l'état de confiance. Enfin, il est exigé qu'aucune période de transition ne soit prévue pour que le particulier s'adapte au nouveau comportement de la personne morale publique et que ses droits ou intérêts particuliers ne soient pas affectés car, si ces mesures transitoires ont été adoptées, ou comme c'est le cas lorsque le droit futur est directement reconnu lorsque les dispositions antérieures sont maintenues, il n'y a pas de préjudice.

Selon ces conditions, la responsabilité extracontractuelle de l'État législateur ne se limite pas aux cas dans lesquels il existe des droits consolidés, mais aussi aux cas dans lesquelles sont affectés des espérances ou des attentes légitimes, et c'est en ce sens que le nouveau titre d'imputation de la responsabilité de l'État a été introduit dans les termes suivants :

Le titre d'imputation par excellence à l'encontre de la violation de droits acquis ou de situations juridiquement consolidées, constitutives de dommages-intérêts à l'occasion d'une loi déclarée exécutoire, est le dommage spécial, alors que, en ce qui concerne l'affectation des attentes légitimes et des états de confiance, le résultat dommageable est imputé à la méconnaissance du principe de confiance légitime. (Conseil d'État Colombien, Arrêt du 31 août 2015)

Via Inveniendi Et Iudicandi e-ISSN: 1909-0528 | DOI: https://doi.org/10.15332/19090528 
Cette forme d'attribution de responsabilité correspond spécifiquement aux événements dans lesquels les attentes ou les espérances légitimes ont été trompées, sur la base du principe de protection de la confiance légitime. Néanmoins, il convient de préciser que celui-ci résulte des actions régulières de l'État, c'est-à-dire qui sont conformes au droit, et constitue donc une spécificité de ce qui était précédemment traité par le biais du dommage spécial ou de la rupture de l'équilibre devant les charges publiques. Dans ces conditions, si l'action qui affecte l'espérance légitime est irrégulière, comme cela a été énoncé, elle entraîne l'obligation de réparer les dommages mais en raison de la faute du service.

En ce sens, s'il est vrai que le Conseil d'État a adopté cette nouvelle forme d'imputation de responsabilité pour violation des espérances légitimes, à la suite des actes du législateur, il faut préciser qu'il s'applique aux autres activités de l'État, lorsque, dans l'exercice de leurs fonctions, les attentes sont violées.

Dans le système juridique colombien, ce titre d'imputation a constitué une nouveauté, car si la norme qui générait l'attente était valide et par son altération, il en résultait un préjudice. L'obligation de réparer les dommages causés était donc fondée sur la théorie du dommage spécial ou la rupture de l'égalité devant les charges publiques. Cependant, on utilise maintenant l'attribution spécifique de la responsabilité pour la violation des espérances légitimes fondée sur le principe de la confiance légitime. Une circonstance qui établit l'obligation de l'État de réparer le préjudice causé à l'individu à qui l'espérance légitime a été trompée ; réparation qui est déterminée par l'affectation patrimoniale subie par l’individu, qui constitue un intérêt en cours de consolidation, ce qui permet d'affirmer qu'il existe un vaste champ de protection des droits futurs, au-delà des droits consolidés. 
En effet, en réparant le dommage futur, le juge protège des intérêts particuliers en cours de formation. Cette circonstance semblerait être en contradiction avec les droits subjectifs consolidés, dans la mesure où les droits futurs ne sont pas entrés dans le patrimoine de la personne, de sorte qu'il ne serait pas possible de les rétablir puisque, dans le système juridique colombien, la théorie qui a été appliquée est celle de l'intérêt lié à la réparation des préjudices, qui consiste à comparer le patrimoine d'une personne avant et après le fait générateur de l'affectation, la différence serait l'intérêt pour la réparation. Pourtant, comme dans ce cas, le droit n'est pas entré dans le patrimoine de l'individu. On ne pourrait donc pas faire une telle comparaison.

Malgré ce qui a été mentionné, la jurisprudence a adopté l'approche suivante : même si le droit n'est pas encore inscrit dans le patrimoine de l'individu, il existe une forte probabilité, une attente sérieuse qu'il entre dans le patrimoine. Pour cette raison, il est protégé et réparé ; à cet égard, le Conseil d’État a déclaré :

Il s'agit de situations visant à la formation d'un droit subjectif, formées par les attentes légitimes découlant de l'exercice de l'autonomie de la volonté privée exprimée dans la constitution des faits prévus par la loi pour l'acquisition du droit et que, bien qu'ils n'aient pas encore entré le droit dans le patrimoine du sujet, ils offrent la certitude que le parcours des faits juridiques sera constitué par le droit. Ce sont donc des situations qui, bien qu'elles ne soient pas consolidées et n'aient pas créées une situation d'acquisition d'un droit, elles ont créé des attentes valables, fondées sur la réalisation progressive des cas de fait légalement évalués pour l'obtention du droit et bénéficient donc d'une protection. (Conseil d'État Colombien, Arrêt du 31 août 2015)

Comme il s'agit d'une indemnisation pour dommages et intérêts, la règle générale est que les dommages causés soient réparés financièrement, c'est- 
à-dire indemnisés, en faisant valoir que le droit subjectif ne figurait pas dans le patrimoine de la personne, de sorte qu'un droit inexistant ne pouvait pas être restauré comme forme de protection de celui-ci. Cela signifie que la protection de l'espérance légitime prendrait la forme d'une simple compensation financière pour les dommages subis. Cette circonstance a été présentée dans la jurisprudence colombienne, entre autres, en cas de résiliation unilatérale des procédures précontractuelles (Conseil d'État Colombien, Arrêt du 1 avril 2016), d'escroquerie par rapport aux attentes dans les projets d'urbanisme (Conseil d'État Colombien, Arrêt du 5 juillet 2018), de modification des plans d'aménagement du territoire, entre autres (Conseil d'État Colombien, Arrêt du 29 de février 2016).

\section{Conclusions}

1. Il existe toutefois différentes garanties permettant la protection des intérêts individuels, comme la sécurité juridique, même s’il s'agit d'une institution ayant un fondement objectif, il est évident que sa finalité est la protection des situations juridiques individuelles telles que les droits acquis, mais qui peut être étendue pour garantir la protection de droits futurs ou en cours de consolidation.

2. En droit colombien, la confiance légitime est un principe qui régit toute l'activité de l'État et qui a été reconnu comme une institution juridique se situant à mi-chemin entre les droits reconnus et un simple sentiment. Cela consiste alors dans la conviction, la croyance, le degré de certitude ou de haute probabilité que le but poursuivi dans une action étatique sera atteint, un but qui se concrétise par l'acquisition d'un droit futur ou en cours de consolidation. Cela veut dire qu'il entrera très certainement dans le patrimoine d'un individu. 
3. Dans certains cas, les espérances légitimes sont violées et il est impossible d'atteindre le but recherché, ce qui crée une obligation de garantie et de protection.

4. Les espérances légitimes peuvent être protégées de diverses manières et les mécanismes varient dans le système juridique français et colombien, mais la réparation économique des dommages est la forme la plus courante de protection.

5. C'est pourquoi, en Colombie, il a été établi un titre d'imputation de la responsabilité de l'État pour la violation des attentes légitimes, sur la base de l'atteinte au principe de la confiance légitime, contrairement aux formes traditionnelles d'attribution de la responsabilité qui étaient données dans ce cas, il s'agissait de l'faute du service et de la responsabilité pour dommage spécial.

6. Dans le système juridique français, la protection des espérances légitimes trouvent un fondement dans les critères classiques de responsabilité tels que la rupture de l'égalité devant les charges publiques.

7. Enfin, il convient d'indiquer que, dans le cas particulier de la notion d'espérance légitime, le droit colombien a connu un développement plus large que le système juridique français, où il y a eu quelques restrictions de sorte qu'il a été nécessaire de faire appel aux règles du droit communautaire et de la convention européenne des droit de l'homme pour sa reconnaissance.

\section{Références Bibliographique}

\section{Ouvrages}

Broyelle, C. (2009). Confiance légitime et responsabilité publique. Revue du Droit Public, 2,321 . 
Broyelle, C. (10 avril 2018). Responsabilité du fait de l'activité normative de l'État et protection des attentes légitimes. En Colloque La protection des attentes légitimes en droit international, en droits européens et en droit public interne. Le Mans Université : Thémis.

Calmes, S. (2001). Du principe de protection de la confiance légitime en droits allemand, communautaire et français. Dalloz.

Calmes, S. (2015). De la protection Constitutionnelle de l'intérêt public général à celle des attentes légitimes des personnes. En A. Cartier-Bresson, M. Collet et C. A. Dubreuil, L’intérêt général - Mélanges en l'honneur de Didier Truchet (pp. 53-66). Dalloz.

Collet, M. (2017). La loyauté en droit fiscal. En S. Niquege, Les figures de la loyauté en droit public. Mare \& Martin.

De Cupis, A. (1970). El daño: teoría de la responsabilidad civil. Bosch.

Debré, J. L. (24 janvier 2014). Discours introductif. En Colloque sur la QPC. Conseil Constitutionnel.

Deguergue, M. (1994). La Jurisprudence et doctrine dans l'élaboration du droit de la responsabilité administrative. LGDJ.

Dupuis, G. et Guédon, M. J. (2004). Droit Administratif (9e éd.). Armand Colin.

Hernández Valbuena, G. (2007). La defraudación de la confianza legítima. Universidad Externado de Colombia.

Jean-Baptiste, W. (2011). L'espérance légitime. Fondation Varenne (Collection de Thèses).

Plessix, B. (2016). Sécurité juridique et confiance légitime. Revue du Droit Public, 3, 799.

Rougevin-Baville, M. (1992). La responsabilité administrative. Hachette.

Saavedra Becerra, R. (2005). La responsabilidad de la Administración Pública. Ibáñez.

Salle, F. (1994). La perte de chance dans la jurisprudence administrative relative à la responsabilité de la puissance publique. LGDJ.

Sänger, R. (2015). La garantía de la propiedad y el principio de proporcionalidad como límites de la carga tributaria en Alemania en Revista IUSTA, (42), 73-99.

https://doi.org/10.15332/s1900-0448.2015.0042.04

Via Inveniendi Et Iudicandi

e-ISSN: 1909-0528 | DOI: https://doi.org/10.15332/19090528

Vol. 16 N.० 2 | julio-diciembre del 2021 
Truchet, D. (2019). Droit Administratif ( $8^{\mathrm{e}}$ éd. mise à jour). Thémis.

Vargas Florián, S. M. (2018). La fuerza vinculante de la jurisprudencia del Consejo de Estado. IUSTA, (48), 119-144. https://doi.org/10.15332/s1900-0448.2018.0048.05

Yannakopoulos, C. (1997). La notion de droits acquis en droit administratiffrançais. Dalloz.

\section{Jurisprudence}

Conseil d'État Colombien. (1 avril 2016). Arrêt n $n^{\circ}$ 11001-O3-26-Ooo-2011-Ooo28oo(41217). Rapporteur : Jaime Orlando Santofimio Gamboa.

Conseil d'État Colombien. (11 août 2010). Arrêt $n^{\circ}$ 18593. Rapporteur : Mauricio Fajardo Gómez.

Conseil d'État Colombien. (14 mars 2019). Arrêt n ${ }^{\circ}$ 20001-23-31-OOO-2011-OO457-O1 (48635). Rapporteur : María Adriana Marín

Conseil d'État Colombien. (15 novembre 2011). Arrêt $n^{\circ} 23001233100019970893401$. Rapporteur : Olga Mélida Valle De La Hoz.

Conseil d'État Colombien. (17 mai 2012). Arrêt n $n^{\circ} 11001-03-25-000-2009-00141-$ oo(2120-o9) et Arrêt n ${ }^{\circ}$ 11OO1-03-25-OOO-2009-OO146-OO(2125-09). Rapporteur : Víctor Hernando Alvarado Ardila.

Conseil d'État Colombien. (23 mai 2012). Arrêt n ${ }^{\circ}$ 17001-23-3-1000-1999-0909o1(22592). Rapporteur : Enrique Gil Botero.

Conseil d'État Colombien. (24 janvier 2019). Arrêt $n^{\circ} 76001233100020080029001$ (41705). Rapporteur : Ramiro Pazos Guerrero.

Conseil d'État Colombien. (28 janvier 2015). Arrêt nº 05001233100020020348701 (32912). Rapporteur : Jaime Orlando Santofimio Gamboa.

Conseil d'État Colombien. (29 de février 2016). Arrêt n ${ }^{\circ}$ O7007-23-31-OOO-2003-OOO1501 (35505).

Conseil d'État Colombien. (31 août 2015). Arrêt n ${ }^{\circ}$ 2500o-23-26-ooo-1999-Oooo7o1(22637). Rapporteur : Ramiro de Jésus Pazos Guerrero.

Conseil d'État Colombien. (31 août 2015). Arrêt $n^{\circ}$ 22637. Rapporteur : Ramiro Pazos Guerrero.

Via Inveniendi Et Iudicandi

e-ISSN: 1909-0528 | DOI: https://doi.org/10.15332/19090528

Vol. 16 N.o 2 | julio-diciembre del 2021 
Conseil d'État Colombien. (31 mars 2016). Arrêt n ${ }^{\circ}$ 11001-03-15-OoO-2016-OO4O2$o o(A C)$. Rapporteur : William Hernández Gómez.

Conseil d'État Colombien. (5 avril 2017). Arrêt n 17001-23-31-Ooo-20oo-oo645-o1. Rapporteur : Ramiro Pazos Guerrero

Conseil d'État Colombien. (5 juillet 2018). Arrêt n ${ }^{\circ}$ 76001-23-31-OoO-2002-04551o1(38942). Rapporteur : Jaime Orlando Santofimio Gamboa.

Conseil d'État, 4/1 SSR. (27 avril 1983). Requête no 20648. Ministre de L’Interieur. Societé Sirap.

Conseil d'État, Assemblée. (24 décembre 2019). Requête $n^{\circ}$ 425981. Société Paris Clichy.

Conseil d'État, Assemblée. (24 décembre 2019). Requête $n^{\circ} 425983$.

Conseil d’État, Assemblée. (24 décembre 2019). Requête $n^{\circ} 428162$.

Conseil d'État. (12 novembre 12). Requête $n^{o} 62342$.

Conseil d'État. (13 mai 1987). Alderbert.

Conseil d'État. (14 janvier 1938). Requête $n^{\circ}$ 517041938. Société des produits laitiers La Fleurette.

Conseil d'État. (1 ${ }^{\mathrm{er}}$ mars 1940). Société Chardon et Compagnie, Lebon.

Conseil d'État. (2 novembre 2005). Requête $n^{\circ}$ 266564. Société Coopérative Agricole Ax'ion.

Conseil d'État. (22 février 1963). Commune de Gavarnie.

Conseil d'État. (24 avril 1953). Etablissements Chosson, Lebon.

Conseil d'État. (24 avril 1964). A propos de la promesse d'octroyer un contingent d'exportation a une société sur une durée déterminé. Société des huileries de Chauny.

Conseil d’État. (26 octobre 1973). Requête no 87909. SCI Résidence Arcole.

Conseil d’État. (29 décembre 2004). Almirayac.

Conseil d’État. (29 octobre 1976). Dame Burgat.

Conseil d'État. (30 mars 1966). Compagnie générale d'énergie radio-électrique.

Conseil d'État. (30 novembre 1923). Requête $n^{\circ} 3828448688$. Couitéas.

Via Inveniendi Et Iudicandi

e-ISSN: 1909-0528 | DOI: https://doi.org/10.15332/19090528

Vol. 16 N.o 2 | julio-diciembre del 2021 
Conseil d'État. (6 décembre 2017). Requête no 400406.

Cour Constitutionnelle Colombienne. ( $1^{\mathrm{er}}$ juillet 2009). Décision C-428. Rapporteur : Mauricio González Cuervo.

Cour Constitutionnelle Colombienne. (7 septembre 2011). Décision T-662 de 2011.

Rapporteur : Jorge Ivan Palacio Palacio.

Cour de Cassation, Chambre Criminelle. (6 juin 1990).

Via Inveniendi Et Iudicandi

e-ISSN: 1909-0528 | DOI: https://doi.org/10.15332/19090528

Vol. 16 N.0 2 | julio-diciembre del 2021 